\title{
Research of inhibition of survivin in rat HSC-T6 cell by siRNA interference
}

\author{
Yang $\mathrm{MF}^{1}, \mathrm{Li} \mathrm{DF}^{2}, \mathrm{Nie} \mathrm{YQ}^{2}$ \\ Zhao Qing No.1 People’s Hospital, Zhaoqing, PR China. Idf830712@163.com
}

\begin{abstract}
OBJECTIVE: To explore the effects and mechanisms of siRNA targeting survivin of inducing apoptosis in rat HSC-T6 cells.

METHODS: The experiment was divided into blank group, pGPU6/GFP/Neo-shNC group and pGPU6/GFP/ Neo-siRNA group. The siRNA was transfected into HSC-T6 cells mediated by Lipofectamine ${ }^{\mathrm{TM} 2000}$ for $24 \mathrm{~h}$, and then the efficiency of transfection was observed by fluorescence microscopy. After transfection for $48 \mathrm{~h}$, the expression of survivin mRNA and protein was assessed by reverse transcription-polyme-rase chain reaction (RT-PCR) method and Western-blot, and the form of cells was observed by microscopy. The apoptosis rate of HSC-T6 cells was measured by the flow cytometry with PI staining. The expression of caspase-3 protein was assessed by western-blot.

RESULTS: The prominent apoptosis of the pGPU6/GFP/Neo-siRNA group by PI staining was high, there was significant difference comparing with blank group and pGPU6/GFP/Neo- shNC group $(p<0.05)$. The prominent apoptosis of blank group and pGPU6/GFP/Neo-shNC group was weak, there was no significant difference among blank group and pGPU6/GFP/Neo-shNC group ( $p>0.05$ ). The expression of caspase-3 by Westernblot in pGPU6/GFP/Neo-siRNA group was high, there was significant difference comparing with blank group and pGPU6/GFP/Neo- shNC group ( $<0.05$ ), the expression of caspase-3 in blank group and pGPU6/GFP/ Neo- shNC group was weak, there was no significant difference among blank group and pGPU6/GFP/NeoshNC group $(p>0.05)$.

CONCLUSION: SIRNA targeting survivin can inhibit the expression of survivin mRNA and protein in rat HSC-T6 cells. Expression of survivin is negative correlation with expression of caspase-3. siRNA targeting survivin may upregulate expression of caspase-3 and increase apoptosis of rat HST-T6 (Fig. 6, Ref. 24). Text in PDF www.elis.sk. KEY WORD: survivin, siRNA, hepatic stellate cell, caspase-3, apoptosis.
\end{abstract}

\section{Introduction}

Hepatic fibrosis (HF) is an inevitable stage of the progression of various chronic hepatic diseases. It is the key to the cure of chronic hepatic diseases and to reversing pathological changes at HF stage (1). The pathway of the genesis of HF is the activation of the hepatic stellate cell (HSC), which is crucially linked to the formation of HF (2). Among various treatment methods of HF, the HSC comes into focus of research as a major target (2-5). However, there has been found no completely specific gene or receptor molecule on HSC. So far, no major breakthrough has been made in the treatment of HF.

Survivin is a new inhibitor of apoptotic protein (IAP), obtained by Altieri et al in 1997 through isolating hybrid between cDNA

'Zhao Qing No.1 People's Hospital, Zhaoqing, , PR China, and ' ${ }^{\text {Guang- }}$ zhou No.1 People's Hospital, Guangzhou Medical University, Guangzhou, PR China

Address for correspondence: Nie Yu Qiang, M.D, Guangzhou No.1 People's Hospital, Guangzhou Medical University, Guang Zhou, 518000, PR China. Phone: +86.02081048888

Acknowledgements: We sincerely thank Dr Zeng for careful language editing of the manuscript. of effector cell protease receptor 1 (EPR-1) and that in the human genomic library (6). It is the strongest inhibitor of apoptosis protein that has been ever found so far. As opposed to other IAP family members, the survivin gene expression has clear tissue selectivity. It generally has high expression in embryonic tissues and malignant tumors, whereas it is not expressed in differentiated and mature tissues (except for trace amount of expression in placenta, endometrium in proliferation period and secretion period) and in paracancerous normal tissues (7). As a new member, IAP is considered an ideal target for genetic treatment of tumors due to its tissue selectivity and unique biological functions $(8,9)$.

De et al (10) found that static HSC had no survivin gene expression. In animal model for activated HSC in culture, bile duct ligation or nitrogen tetrachloride treatment, the survivin gene expression had a significant increase. Minicis DS et al found that survivin had high expression in activated HSC (11). Therefore, survivin might be correlated with the genesis and development of hepatic fibrosis.

Caspase-3 belongs to the interleikin-1 converting enzyme (ICE) family as its most important member. It is an apoptotic proteinase. In normal conditions, the caspase- 3 in the cytoplasm exists in its original form as an inactive enzyme. Once activated, 
the cells undergo apoptosis (12). Research indicates that caspase-3 induces cell apoptosis via mechanism as follows: (1) inhibition or inactivation of protein during the DNA formation; (2) inhibition of inhibitor of apoptosis protein; (3) cutting of structural proteins of the cell (13). During their research on induction of cell apoptosis, Fas, Cui JH et al indicated the activation of caspase- 3 and that the serine proteinase inhibitor could inhibit the activation of caspase- 3 and cell apoptosis simultaneously (14). In addition, studies also found that after activation, caspase- 3 could cause cell apoptosis with the amplification effect of proteinase cascade. Therefore, caspase-3 is called cell-death proteinase (15). Therefore a question is to be raised, namely whether the activated HSC expresses the survivin gene and inhibits the expression of caspase-3, while the process finally results in the inhibition of HSC apoptosis.

RNA interference technology is a specific gene inactivation technology, which introduces double-strand RNA into the cell and under the effect of Dicer enzyme it produces active molecules to interfere with RNA fragments. In this way, it causes specific degradation of mRNA homogeneous to RNA fragment and inhibits the expression of the corresponding genes, so as to produce the effect similar to gene knockdown (16). With the strong effect, stability and persistence as well as the transmission of the effects to the filial generation, the RNA interference technology is widely applied in the studies of genetic functions and treatment of tumors (17-18). This research uses RNA interference technology to produce the targeted effects on survivin gene, so as to study the influence on HSC apoptosis and caspase-3 gene. The work provides a theoretical foundation for the treatment of hepatic fibrosis.

\section{Methods and materials}

HSC-T6 cells were brought from South China University. HCS-T6 cells were incubated in culture medium with $5 \% \mathrm{CO}_{2}$ in an incubator (SANYO company, Japan), according to the manufacturer instructions for indicated period of time after replacement of transfection culture medium in DMEM with $5 \%$ FBS. Then, HCS-T6 cells were collected by Centrifuge (Eppendorf Company, Germany). HCS-T6 cells were divided into blank group (not transfected), pGPU6/GFP/Neo-shNC group (transfecting pGPU6/GFP/ Neo-shNC, Zimmer Biological Company, Shanghai, China) and pGPU6/GFP/Neo-siRNA group (transfecting pGPU6/GFP/NeosiRNA, Zimmer Biological Company, Shanghai, China). The siRNA was transfected into cells mediated by LipofectamineTM2000 for $24 \mathrm{~h}$, according to the manufacturer instructions, and then the efficiency of transfection was observed by fluorescence microscopy (Olympus Company, Japan). After $48 \mathrm{~h}$ following transfection, the expression of survivin mRNA was assessed by reverse transcription-polymerase chain reaction (RT-PCR) method. Total RNA was extracted by Trizol reagent (Invitrogen). Reaction mixture $(20 \mu \mathrm{l})$ contained RNA $(2 \mu \mathrm{l})$, oligo(dT) $18(1 \mu \mathrm{l})$, Reaction Buffer $(4 \mu \mathrm{l})$, RiblockTM RNase inhibition $(1 \mu \mathrm{l}), 10 \mathrm{Mm}$ dNTP Mix $(2 \mu \mathrm{l})$, RevertAidTM M-Mulv Reverse Transcriptase $200 \mu$ / $\mu \mathrm{l}(1 \mu \mathrm{l})$, DEPC-treated water $(9 \mu 1)$. The PCR conditions were as follows: activation of enzyme at $94{ }^{\circ} \mathrm{C}$ for $4 \mathrm{~min}, 35$ circles of denaturation at $94{ }^{\circ} \mathrm{C}$ for $30 \mathrm{~s}$, annealing at $57{ }^{\circ} \mathrm{C}$ for $30 \mathrm{~s}$, and extension at $72{ }^{\circ} \mathrm{C}$ for $30 \mathrm{~s}$. Relative expression of survivin mRNA was calculated as ratios normalized against those of $\beta$-action. The expression of survivin protein and caspase-3 protein was assessed by Western-blot. Cells were harvested and lysed in $1.0 \mathrm{ml}$ of lysis buffer. Lysates were clarified by centrifugation at $12000 \mathrm{r} / \mathrm{min}$ for $10 \mathrm{~min}$. Protein was processed for SDS-PAGE, which was performed on $12 \%$ gels. The blots were blocked with $5 \%$ non-fat milk in Tris-buffered saline for $2 \mathrm{~h}$ and then incubated with antibodies in $5 \%$ non-fat milk in TBS, then washed with TBS and incubated with secondary antibodies conjugated with horseradish peroxidase in $5 \%$ non-fat milk in TBS. After washing with TBS, the bound antibodies were visualized by enhanced chemiluminescence and recorded on X-ray films. The form of HSC-T6 cells was observed by microscopy (Olympus Company Japan). The apoptosis rate of HSC-T6 cells was measured by flow cytometry with PI staining (FACScan Becton Dickinson, USA ).

\section{Statistical analysis}

Data were expressed as mean \pm SD. Differences with $\mathrm{p}<0.05$ were considered statistically significant. These analyses were performed using SPSS16.0 software.

\section{Results}

\section{Observation of siRNA transfecting into HSC-T6 cells}

After $24 \mathrm{~h}$ following transfection, clear and green fluorescence was observed by inversion fluorescence microscope in HSC-T6, and the efficiency of transfection was attained to $75 \%$ (Fig. 1).

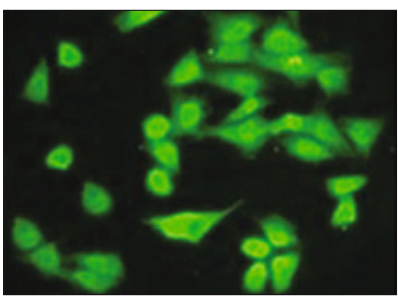

PGPU6/GFP/Neo-shNC group

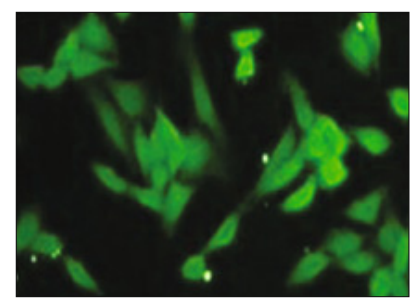

pGPU6/GFP/Neo-siRNA group
Fig. 1. PGPU6/GFP/Neo-shNC group and pGPU6/GFP/Neo-siRNA group.
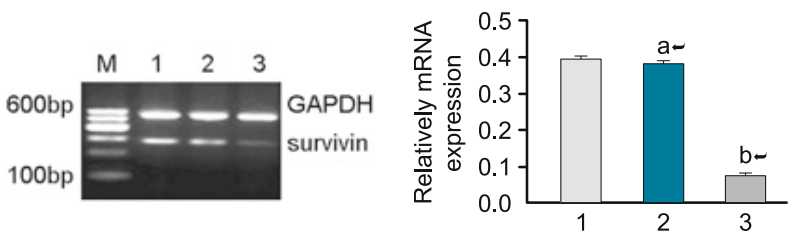

Fig. 2. Relatively mRNA-expression.
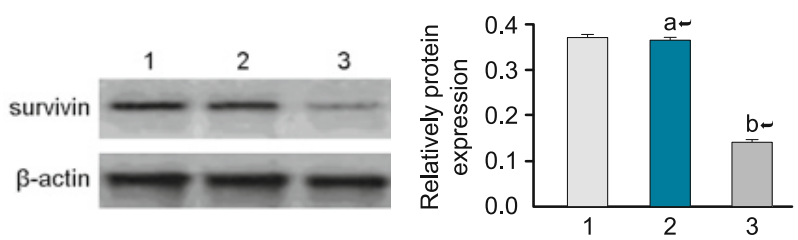

Fig. 3. Relatively protein-expression. 


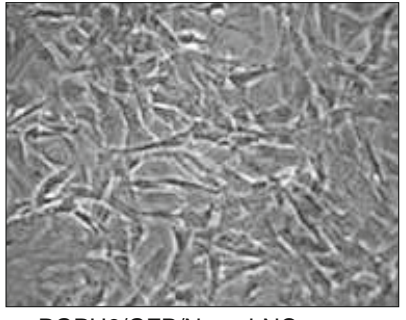

PGPU6/GFP/Neo-shNC group

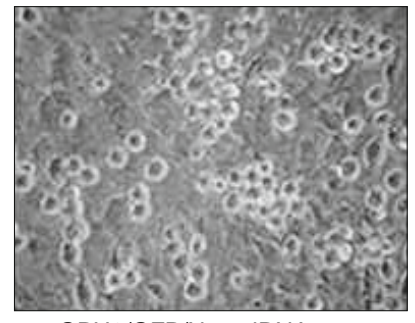

pGPU6/GFP/Neo-siRNA group
Fig. 4. pGPU6/GFP/Neo-shNC group and pGPU6/GFP/Neo-siRNA group.

Expression of survivin mRNA in siRNA transfection of HSC-T6

The expression of survivin mRNA in HSC-T6

The expression of survivin mRNA in blank group and pGPU6/ GFP/Neo-shNC group by RT-PCR was higher; there was no significant difference between blank group and pGPU6/GFP/Neo- shNC group $(\mathrm{p}>0.05)$. The expression of survivin mRNA in pGPU6/ GFP/Neo-siRNA group was obviously weak; there was a significant difference when compared with blank group and pGPU6/ GFP/Neo- shNC group ( $<$ 0.05) (Fig. 2).

The expression of survivin protein in HSC-T6

The expression of survivin protein in blank group and pGPU6/ GFP/Neo-shNC group by Western blotting was higher; there was no significant difference between blank group and pGPU6/GFP/ Neo- shNC group $(\mathrm{p}>0.05)$. The expression of survivin protein group was obviously weak; there was significant difference when compared with blank group and pGPU6/GFP/Neo- shNC group $(\mathrm{p}<0.05)$ (Fig. 3).

\section{Apoptosis of siRNA transfection of HSC-T6}

Transformation of HSC-T6

When observed under microscope $48 \mathrm{~h}$ after transfection of HSCT6 cells, HSC-T6 cells were adhering to the wall, had normal morphology, and were in a vigorous growth stage in pGPU6/GFP/NeoshNC group. In pGPU6/GFP/Neo-siRNA group, whereas, HSC-T6 cells were suspended from the wall, they were shrunk, rounded, their number decreased, and they yielded slow growth (Fig. 4).
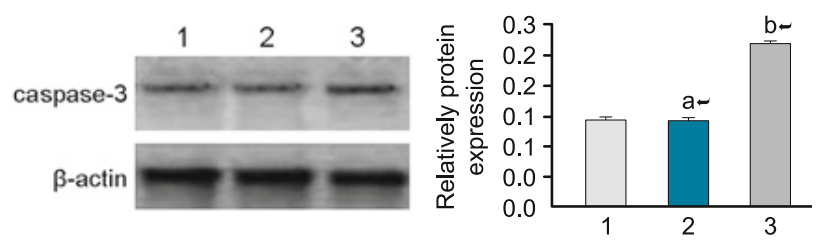

Fig. 6. Relatively protein-expression.

Apoptosis rate of HSC-T6 cells by the flow cytometry

When cells became apoptotic because of cellular DNA content by flow cytometry, there was a feature, in which the left of diploid peak (G1 peak) exhibited sub-diploid karyotype peak (Ap peak). In blank group and pGPU6/GFP/Neo-shNC group, there was no Ap peak. However, in pGPU6/GFP/Neo-siRNA, there was an obvious AP peak, which showed that the apoptosis rate of pGPU6/GFP/Neo-siRNA group was higher than those in blank group and pGPU6/GFP/Neo-shNC group at a significant difference $(\mathrm{p}<0.05)$ (Fig. 5).

\section{The expression of caspase-3 protein in HSC-T6}

The expression of caspase- 3 protein in blank group and pGPU6/GFP/Neo-shNC group by Western blot was weak, there was no significant difference between blank group and pGPU6/ GFP/Neo- shNC group $(\mathrm{p}>0.05)$. The expression of caspase-3 protein in pGPU6/GFP/Neo-siRNA group was obviously higher; there was a significant difference when compared with blank group and pGPU6/GFP/Neo- shNC group $(\mathrm{p}<0.05)$ (Fig. 6).

\section{Discussion}

HF is a wound-healing response to hepatic damage caused by various reasons by the liver itself. It is manifested as hyperplasia and deposition of connective tissues in the liver, which is an inevitable stage of the progression of various chronic hepatic diseases to hepatic cirrhosis. In clinical practice, the question whether the pathological changes can be reversed at the HF stage is considered to be the key to the cure of chronic hepatic diseases (1). The pathologic mechanisms of chronic hepatic damage differ based on

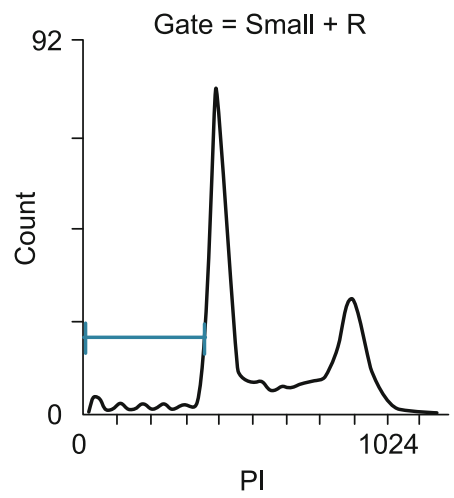

pGPU6/GFP/Neo-shNC group

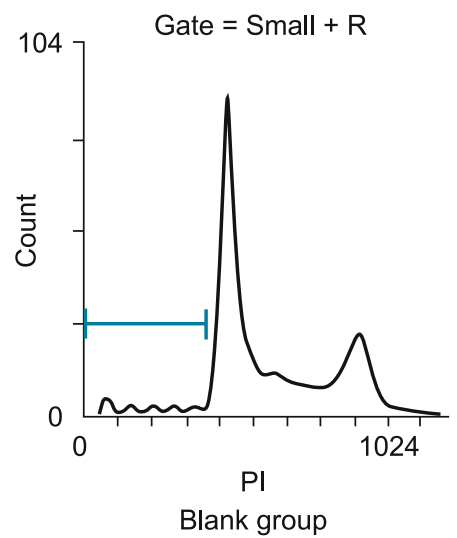

Blank group

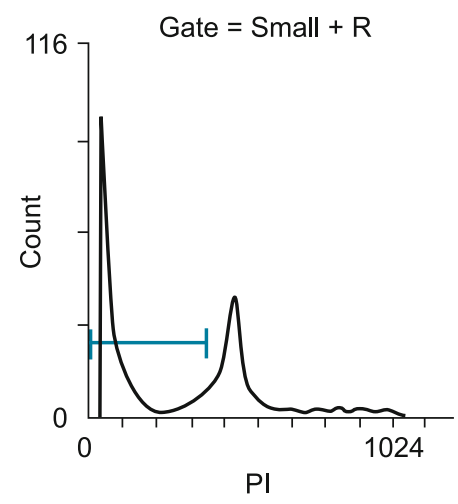

pGPU6/GFP/Neo-siRNA group

Fig. 5. pGPU6/GFP/Neo-shNC group, blank group and pGPU6/GFP/Neo-siRNA group. 
the variability of causes. However, the final pathway of HF genesis is the activation of HSC. The activated HSC can synthesize a large amount of collagen (except for type $\mathrm{V}$ collagen), hyaluronic acid, laminin, fibronectin, etc. and participates in the synthesis and regulation of collagen and its inhibitors (2-5). The amount of collagen synthesized by hepatic stellate cells is more than ten times greater than that produced by hepatic cells and over 20 times greater than that produced by sinusoidal endothelial cells. HSC also impedes the degradation of ECM and activates further static HSC by secreting tissue inhibitor of metalloproteinase (TIMPs) (18) and transforming growth factor $\beta$ (TGF- $\beta$ ) (19), which forms a vicious circle difficult to be reversed. The explanation of HSC activation mechanism has turned HSC into an important target in the effort of curing HF (20). The search for specific gene or receptor molecule of HSC is crucial for the treatment of hepatic fibrosis.

Cationic liposome is a new liposome with cation-mediated transfection. It has a unique structure of strongly negative electronic head of the spermine gene, which can form spontaneously complexes with DNA with strong transfection ability. Moreover, it is safe for cells because it has no toxic effects. The study shows that $24 \mathrm{~h}$ following transfection of siRNA with cationic liposome, green fluorescence in pGPU6/GFP/Neo-shNC and pGPU6/GFP/ Neo-siRNA cells can be observed under the inverted fluorescence microscope. The transfection efficiency can reach approx. $75 \%$. The research provides a solid foundation for further studies.

Minicis found that survivin had high expression in activated HSC (10). In addition, survivin is the strongest apoptosis inhibitor that has been ever known. It enhances the abnormal proliferation of cells by interfering with mitosis (6-7). As an answer to the question whether siRNA transfection technology inhibits the survivin gene expression in activated HSC, it is found out that the survivin mRNA and protein expressions were the highest in the blank group and pGPU6/GFP/Neo-shNC group, and the survivin mRNA and protein expressions were the lowest in pGPU6/GFP/ Neo-siRNA group. The difference between the blank group and the pGPU6/GFP/Neo-shNC group showed no statistical significance $(\mathrm{p}>0.05)$. The difference between the blank group, pGPU6/ GFP/Neo-shNC group and pGPU6/GFP/Neo-siRNA group was statistically significant $(\mathrm{p}<0.05)$. This indicates that siRNA can inhibit survivin gene expression.

In order to answer to the question whether siRNA can promote HSC apoptosis, even though it inhibits survivin gene expression, the morphology of cells after $48 \mathrm{~h}$ of transfection was observed under the inverted microscope and the cell apoptosis rate was measured with flow cytometry. The results indicate that the cell volume in pGPU6/GFP/Neo-siRNA group is decreased, the cells shrink, and the apoptosis increases. When compared with the blank group and pGPU6/GFP/Neo-shNC group, the difference is statistically significant $(\mathrm{p}<0.05)$; the difference between the blank group and pGPU6 /GFP/Neo-shNC group shows no statistical significance $(p>0.05)$. This further proves that survivin gene expression can facilitate HSC apoptosis.

As an answer to the question whether the mechanism that inhibits survivin gene expression and promotes HSC apoptosis is related to caspase- 3 expression, the research found that the cas- pase-3 protein expression was low in the blank group and pGPU6/ GFP/Neo-shNC group; the caspase-3 protein expression was high in pGPU6/GFP/Neo-siRNA group. The difference between the blank group and the pGPU6/GFP/Neo-shNC group showed no statistical significance $(\mathrm{p}>0.05)$; the difference between the blank group, pGPU6/GFP/Neo-shNC group and pGPU6/GFP/ Neo-siRNA group showed statistical significance $(\mathrm{p}<0.05)$. The survivin expression was inhibited and caspase-3 expression was promoted. Survivin expression is in negative correlation with caspase-3 expression. Wang JG's study on squamous cell carcinoma of tongue showed that the survivin expression was in negative correlation with caspase-3 expression (21). Therefore, survivin possibly inhibits caspase-3 expression, thus resulting in obstructed cell proliferation and apoptosis (22). Chu X found that the treatment of rectal cancer by inhibiting survivin gene expression with siRNA interference technology could enhance the expression of pro-apoptotic gene caspase-3 (23). Jeffrey C discovered that ET-1 modulates myofibroblasts susceptibility to apoptosis by increasing Survivin expression while inhibiting expression of survivin can trigger apoptosis in idiopathic pulmonary fibrosis (IPF) (24). These facts are consistent with our study. Therefore, survivin could inhibit caspase-3 expression while impeding the HSC-T6 apoptosis, and thus cause HF; however, siRNA targeting survivin in the HSCT6 can increase expression of caspase- 3 and promote apoptosis, which may be proved an effective way to cure HF.

However, hepatic fibrosis could result from multiple factors and pathways. The question whether other factors or pathways can also cause hepatic fibrosis needs to be studied further.

\section{Learning points}

The siRNA targeting survivin in HSC-T6 can increase expression of caspase- 3 and promote apoptosis, which may prove an effective way to cure hepatic fibrosis.

\section{References}

1. Albanis E, Friedman SL. Hepatic fibrosis pathogenesis and principles of therapy. Clin Liver Dis 2001; 5: 315-334.

2. Eng FJ, Friedman SL. Fibrogenesis I new insights into hepatic stellate cell activation: the simple becomes complex. Am J Physiol 2000; 279: G7-G11.

3. Gressner OA, Weiskirchen R, Gressner AM. Evolving concepts of liver fibrogenesis provide new diagnostic and therapeutic options. Comp Hepatol; 2007: 6-7.

4. Friedman SL. Molecular regulation of hepatic fibrosis, an integrated cellular response to tissue injury. Biol Chem 2000; 275: 2247-2250.

5. Gressner, AM, Weiskirchen, R. Modern pathogenetic concepts of liver fibrosis suggest stellate cells and TGF-beta as major players and therapeutic targets. Cell Mol Med 2006; 10: 76-99.

6. Ambrosini G, Adida C, Altieri DC. A novel anti-apoptosis gene, survivin, expressed in cancer and lymphom. Nat Med 1997; 3: 917-921.

7. Suzuki A, Ito T, Kawano H. survivin initiates procaspase 3/p21 compleform- ation as a result of interaction with Cdk4 to resist Fas-mediated cell death. Oncogene 2000; 19: 1346-1353. 
$446-450$

8. Reed JC. The survivin saga goes in vivo. Clin Invest 2001; 108: 965969.

9. Kobayashi K, Hatano M, Ogasawara T. Expression of a murine homologue of the inhibitor of apoptosis protein is related to cell proliferation. Proc Natl Acad Sci USA 1999; 96: 1457-1462.

10. Minicis DS, Seki E, Uchinami H. Gene expression profiles during hepatic stellate cell activation in culture and in vivo. Gastroenterology, 2007; 132: 1937-1946.

11. Lee UE, Friedman SL. Mechanisms of hepatic fibrogenesis. Best Pract Res Clin Gastroenterol 2011; 25: 195-206.

12. HU Hui hua, ZHOU, Shi fu, LI Qing guo. Expression of caspase-7, caspase-3, survivin in breast neoplasm and their relationship with clinicpathologic factor. Mod Oncol 2007; 115: 640-642.

13. Cui JH, Qiao Q, Guo Y. Increased apoptosis and expression of FasL, Bax and caspase-3 in human lupus nephritis class II and IV. J Nephrol 2011; 25: 255-261.

14. Zhu L, Yuan H, Guo C. Zearalenone induces apoptosis and necrosis in porcine granulosa cells via a caspase-3- and caspase-9-dependent mitochondrial signaling pathway. J Cell Physiol 2011; 227: 1814-1820.

15. Gao Y, Liu XL, Li XR. Research progress on siRNA delivery with nonviral carriers. Int J Nanomed 2011; 6: 1017-1025.

16. Aoki Y, Cioca DP, Oidaira H. RNA interference may be more potent than antisense RNA in human cancer cell lines. Clin Exp Pharmacol Physiol 2003; 30: 96-102.
17. Takahashi Y, Nishikawa M, Takakura Y. In vivo siRNA delivery to tumor cells and its application to cancer gene therapy. Yakugaku Zassh. 2007; 127: 1525-1531.

18. Murphy FR,Issa R, Zhou X. Inhibition of apoptosis of activated hepatic stellate cells by tissue inhibitor of metalloproteinase- 1 is mediated via effects on matrix metalloproteinase inhibition. Biol Chem 2002; 277: 11069-11076.

19. Aki Kaimori, James Potter Kaimori JY. Transforming Growth Factor- $\beta 1$ induces an Epithelial-to-Mesenchymal Transition State in Mouse Hepatocytes in Vitro. Biol Chem 2007; 282: 22089-22101.

20. Friedman SL. Liver fibrosis-from bench to bedside. Hepatol 2003; 38: S38-S53.

21. Zhu L, Yuan H, Guo C. Zearalenone induces apoptosis and necrosis in porcine granulosa cells via a caspase-3 and caspase-9 dependent mitochondrial signaling pathway. J Cell Physiol 2011; 227: 1814-1820.

22. Wang JG, Pan CB, Zhang B. Survivin shRNA induces caspase -3 dependent apoptosis and enhances cisplatin sensitivity in squamous cell carcinoma of the tongue. Oncol Res 2010; 18: 377-385.

23. Lu H, Gan M, Zhang G. Expression of survivin, caspase-3 and p53 in cervical cancer assessed by tissue microarray: correlation with clinicpathology and prognosis. Eur J Gynaecol Oncol 2010; 31: 662-666.

24. Jeffrey C, Horowitza I, Ajayia O, Kulasekarana P. Survivin expression induced by endothelin-1 promotes myo-fibroblast resistance to apoptosis. Intern J Biochem Cell Biol 2012; 44: 158-169. 